\title{
Optimization for sustainable manufacturing based on axiomatic design principles: a case study of machining processes
}

\author{
Lee, G.B. ${ }^{\mathrm{a},}{ }^{*}$, Badrul, . $^{\mathrm{a}}$ \\ ${ }^{a}$ Faculty of Mechanical and Manufacturing Engineering, Universiti Tun Hussein Onn Malaysia, 86400 Parit Raja, Batu Pahat, \\ Johor, Malaysia
}

\begin{abstract}
A B S T R A C T
Despite being a wasteful process, machining is often regarded as an important manufacturing method due to the fact that it is a flexible and economic process. However, in order to gain more cost-saving and enhanced environmental performance, sustainability principles have to be incorporated into machining technologies. A step-wise optimization procedure is proposed based on axiomatic design (AD) principles for identifying an optimized sustainable manufacturing solution that comprises combinations of minimum and maximum levels obtainable within the constraints involved (cutting condition, performance and sustainability). A case study involving three alternative processes (namely conventional machining, high pressure jetassisted machining, and cryogenic machining) is presented for demonstrating the application of the proposed approach, which indicated that the suggested procedure is able to facilitate an optimization process by varying the design parameters (DPs) within a particular sequence. In the case study, a hybrid model consisting of crisp and fuzzy AD analysis techniques was also used for analysing the sustainability performances of the processes being considered. The hybrid model is able to point out the most viable machining process that satisfies all the sustainable functional requirements (FRs) by using information content for indication purposes.
\end{abstract}

\section{ARTICLE INFO}

Keywords:

Axiomatic design

Hybrid model

Optimization

Sustainable manufacturing

Machining

*Corresponding author: hd110141@siswa.uthm.edu.my

(Lee, G.B.)

Article history:

Received 18 August 2013

Revised 22 February 2014

Accepted 27 February 2014 


\section{References}

[1] Fratila, D. (2013). Sustainable manufacturing through environmentally-friendly machining, Green Manufacturing Processes and Systems; Materials Forming, Machining and Tribology, pp 1-21, doi: 10.1007/978-3-642-33792-5 1.

[2] Jayal, A.D., Badurdeen, F., Dillon, Jr., O.W., Jawahir, I.S. (2010). Sustainable manufacturing: modeling and optimization challenges at the product, process and system levels, CIRP Journal of Manufacturing Science and Technology, Vol. 2, No. 3, 144-152, doi: 10.1016/j.cirpj.2010.03.006.

[3] Pusavec, F., Kramar, D., Krajnik, P., Kopac, J. (2010). Transitioning to sustainable production - part II: evaluation of sustainable machining technologies, Journal of Cleaner Production, Vol. 18, No. 12, 1211-1221, doi: 10.1016/ j.jclepro.2010.01.015.

[4] Jovane, F., Westkämper, E., Williams, D. (2009). The manufuture road, Springer-Verlag, Berlin Heidelberg.

[5] Gutowski, T., Murphy, C., Allen, D., Bauer, D., Bras, B., Piwonka, T., Sheng, P., Sutherland, J., Thurston, D., Wolff, E. (2005). Environmentally benign manufacturing: observations from Japan, Europe and the United States, Journal of Cleaner Production, Vol. 13, No. 1, 1-17, doi: 10.1016/j.jclepro.2003.10.004.

[6] Wanigarathne, P.C., Liew, J., Wang, X., Dillon, Jr., O.W., Jawahir, I.S. (2004). Assessment of process sustainability for product manufacture in machining operations, In: Proceedings Global Conference on Sustainable Product Development and Life Cycle Engineering, Berlin, Germany, 305-312.

[7] Jawahir, I.S., Wanigarathne, P.C., Wang, X. (2006). Product design and manufacturing processes for sustainability, In: Kutz, M. (ed.), Mechanical Engineers' Handbook: Manufacturing and Management, John Wiley \& Sons, 414-443.

[8] Fratila, D. (2010). Macro-level environmental comparison of near-dry machining and flood machining, Journal of Cleaner Production, Vol. 18, No. 10-11, 1031-1039, doi: 10.1016/j.jclepro.2010.01.017.

[9] Pusavec, F., Krajnik, P., Kopac, J. (2010). Transitioning to sustainable production - part I: application on machining technologies, Journal of Cleaner Production, Vol. 18, No. 12, 174-184, doi: 10.1016/j.jclepro.2009.08.010.

[10] Suh, N.P. (1990). The principles of design, Oxford University Press, New York.

[11] Finger, S., Dixon, J.R. (1989). A review of research in mechanical engineering design. Part I: Descriptive, prescriptive, and computer-based models of design processes, Research in Engineering Design, Vol. 1, No. 1, 51-67, doi: $10.1007 / \mathrm{BF} 01580003$.

[12] Kulak, O., Durmuşoğlu, M.B., Kahraman, C. (2005). Fuzzy multi-attribute equipment selection based on information axiom, Journal of Materials Processing Technology, Vol. 169, No. 3, 337-345, doi: 10.1016/j.jmatprotec. 2005.03.030.

[13] Kulak, O., Cebi, S., Kahraman, C. (2010). Applications of axiomatic design principles: a literature review, Expert Systems with Applications, Vol. 37, No. 9, 6705-6717, doi: 10.1016/i.eswa.2010.03.061.

[14] Kulak, O., Kahraman, C. (2005). Fuzzy multi-attribute selection among transportation companies using axiomatic design and analytic hierarchy process, Information Sciences, Vol. 170, No. 2-4, 191-210, doi: 10.1016/i.ins. 2004.02.021.

[15] Shin, M.K., Lee, H.A., Lee, J.J., Song, K.N., Park, G.J. (2008). Optimization of a nuclear fuel spacer grid spring using homology constraints, Nuclear Engineering and Design, Vol. 238, No. 10, 2624-2634, doi: 10.1016/j.nucengdes. 2008.04.003.

[16] Gumus, B., Ertas, A., Tate, D., Cicek, I. (2008). The transdisciplinary product development lifecycle model, Journal of Engineering Design, Vol. 19, No. 3, 185-200, doi: 10.1080/09544820701232436.

[17] Celik, M., Kahraman, C., Cebi, S., Er, I.D. (2009). Fuzzy axiomatic design-based performance evaluation model for docking facilities in shipbuilding industry: the case of Turkish shipyards, Expert Systems with Applications, Vol. 36, No. 1, 599-615, doi: 10.1016/j.eswa.2007.09.055.

[18] Crafoord, R., Kaminski, J., Lagerberg, S., Ljungkrona, O., Wretland, A. (1999). Chip control in tube turning using high-pressure water jet, In: Proceedings of the Institution of Mechanical Engineers, Part B: Journal of Engineering Manufacture, Vol. 213, No. 8, 761-767, doi: 10.1243/0954405991517191.

[19] Wertheim, R., Rotberg, J., Ber, A. (1992). Influence of high-pressure flushing through the rake face of the cutting tool, CIRP Annals-Manufacturing Technology, Vol. 41, No. 1, 101-106, doi: 10.1016/S0007-8506(07)61162-7.

[20] Pusavec, F., Deshpande, A., M'Saoubi, R., Kopac, J., Dillon, Jr., O.W., Jawahir, I.S. (2008). Predictive performance models and optimization for sustainable machining of a high temperature nickel alloy, In: Proceedings of the 3rd CIRP International Conference "High Performance Cutting Conference, HPC 2008", Ireland, 355-364.

[21] Pusavec, F., Deshpande, A., M'Saoubi, R., Kopac, J., Dillon, Jr., O.W., Jawahir, I.S. (2008). Modeling and optimization of the machining of high temperature nickel alloy for improved machining performance and enhanced sustainability, In: Proceedings of 11th CIRP Conference on Modeling of Machining Operations, Gaithersburg, USA, 21-28.

[22] Kramar, D., Kopac, J. (2009). High performance manufacturing aspects of hard-to-machine materials, Advances in Production Engineering \& Management, Vol. 1-2, 3-14. 


\title{
Optimizacija za trajnostno proizvodnjo na osnovi aksiomatskih pravil: študija primera obdelovalnega postopka
}

\author{
Lee, G.B. ${ }^{a,}{ }^{,}$, Badrul, O. ${ }^{a}$ \\ ${ }^{a}$ Faculty of Mechanical and Manufacturing Engineering, Universiti Tun Hussein Onn Malaysia, 86400 Parit Raja, Batu Pahat, \\ Johor, Malaysia
}

\section{POVZETEK}

Kljub temu, da je mehanska obdelava v osnovi potraten postopek, je pomembna proizvodna tehnika, še zlasti zaradi visoke prilagodljivosti in gospodarnosti. Za zmanjšanje stroškov in večjo okoljsko učinkovitost, morajo biti tudi pri mehanskih obdelavah upoštevana načela trajnostnega razvoja. V članku je predstavljen večstopenjski optimizacijski postopek, ki temelji na načelih aksiomatskega oblikovanja (angl. Axiomatic Design - AD) za iskanje optimalne trajnostne proizvodnje. To sestavljajo kombinacije najnižjih in najvišjih ravni vrednosti znotraj podanih omejitev (t). rezalnih pogojev, zmogljivosti in trajnosti). Študija primera, ki je vključevala tri alternativne obdelovalne postopke (konvencionalno obdelavo, visokotlačno obdelavo z vodnim curkom in kriogensko obdelavo), je pokazala, da predlagani postopek lahko olajša proces optimizacije s spreminjanjem parametrov načrtovanja (angl. Design Parameters - DPs) v določenem zaporedju. V študiji primera je bil uporabljen hibridni model, sestavljen iz klasičnih analitičnih tehnik, prav tako pa mehkih tehnik analize aksiomatskega oblikovanja. Hibridni model je sposoben določiti najuspešnejši proces obdelave, ki izpolnjuje vse trajnostne funkcionalne zahteve (angl. Sustainable Functional Requirements - FRs).

\section{PODATKI O ČLANKU}

Ključne besede:

Aksiomatsko oblikovanje

Hibridni model

Optimizacija

Trajnostna proizvodnja

Obdelava

*Kontaktna oseba:

hd110141@siswa.uthm.edu.my

(Lee, G.B.)

Zgodovina članka:

Prejet 18. avgusta 2013

Popravljen 22. februarja 2014

Sprejet 27. februarja 2014 